\title{
Superstar in noncommutative superspace via covariant quantization of the superparticle
}

\author{
Itzhak Bars \\ Department of Physics, USC, Los Angeles, California 90089-0484, USA, \\ and Theory Division, CERN, CH-1211 Geneva 23, Switzerland \\ Cemsinan Deliduman \\ Feza Gürsey Institute, Çengelköy 81220, Istanbul, Turkey \\ Andrea Pasqua and Bruno Zumino \\ Lawrence Berkeley National Laboratory, 1 Cyclotron Rd., Berkeley, California 94720, USA
}

(Received 19 August 2003; published 20 November 2003)

\begin{abstract}
A covariant quantization method is developed for the off-shell superparticle in 10 dimensions. On shell it is consistent with light cone quantization, while off shell it gives a noncommutative superspace that realizes nonlinearly a hidden 11-dimensional super Poincaré symmetry. The nonlinear commutation rules are then used to construct the supersymmetric generalization of the covariant Moyal star product in noncommutative superspace. As one of the possible applications, we propose this new product as the star product in supersymmetric string field theory. Furthermore, the formalism introduces new techniques and concepts in noncommutative (super)geometry.
\end{abstract}

DOI: 10.1103/PhysRevD.68.106006

\section{MOTIVATION: STAR PRODUCT IN SUPERSTRING FIELD THEORY}

The purpose of this paper is to propose the covariant spacetime supersymmetric generalization of the Moyal star product as a first step in constructing supersymmetric string field theory. The motivation for this work is provided by the Moyal star formulation of string field theory (MSFT) [1-4]. On the way to constructing the superstar, we also obtain new results on the quantization of the off-shell superparticle, and on new group theoretical methods for constructing and evaluating star products based on nontrivial (super)Poisson manifolds.

The first proposal of a covariant nontrivial product in superspace was given in the context of purely fermionic supergravity [5], as $\theta^{\alpha} \cdot \theta^{\beta}=C^{\alpha \beta}$, where $C^{\alpha \beta}$ is the charge conjugation matrix. ${ }^{1}$ A later proposal was given in [6] as $\left\{\theta_{\alpha}, \theta_{\beta}\right\}=x^{\mu}\left(\gamma_{\mu}\right)_{\alpha \beta}$. These ideas were motivated by certain aspects of supergravity or supersymmetry and their mysterious origins were not at that time connected to string theory.
PACS number(s): 11.25.Sq, 11.10.Nx, 11.30.Pb

In the recent literature there are other studies of a star product in noncovariant superspace [7-9] (see also $[10,11]$ ) whose origin is background fields in string theory. The star product in MSFT has also a fundamental but different physical origin, namely, string joining. The superstar product we study in this paper is motivated by MSFT, and as required in that context, is super Poincaré invariant, and has a different structure than the previous proposals.

It has been shown that in the language of string field theory the Moyal product is the simplest description of interactions of bosonic strings, corresponding to string joining [1]. To arrive at this description we express the general string field in the space of mixed position-momentum representation of string modes $A\left(\bar{x}, x_{e}, p_{e}\right)$ (instead of purely position representation), where $\bar{x}^{\mu}$ is the string midpoint, and $\left(x_{e}^{\mu}, p_{e}^{\mu}\right)$, with $e=2,4,6, \ldots$, is an equivalent description of the string excitation modes that are compatible with simultaneous observations in first quantized quantum mechanics of the string. ${ }^{2}$ Then the joining of strings is described by com-

\footnotetext{
${ }^{1} C^{\alpha \beta}$ is antisymmetric for $d=(3,4,5) \bmod (8)$, symmetric for $d=(7,8,9) \bmod (8)$ and mixed (i.e. Lorentz singlet occurs in product of opposite chiral spinors) in $d=(6,10) \bmod (8)$. The product $\theta^{\alpha} \cdot \theta^{\beta}=C^{\alpha \beta}$ is not associative, but an associative covariant fermionic Moyal product $\theta^{\alpha} \star \theta^{\beta}=\theta^{\alpha} \theta^{\beta}+\frac{1}{2} C^{\alpha \beta}$ can be constructed in every dimension generally as $A(\theta) \star B(\theta)$, where $\star=\exp \left(-1 / 2 C^{\alpha \beta}\left(\grave{\partial} / \partial \theta^{\alpha}\right)\right.$ $\times\left(\vec{\partial} / \partial \theta^{\beta}\right)$ ), because the star anticommutator $\left\{\theta^{\alpha}, \theta^{\beta}\right\}_{\star}$ is either zero (antisymmetric $C^{\alpha \beta}$ ) or a constant (symmetric or mixed $C^{\alpha \beta}$ ).

${ }^{2}$ The probability amplitude in position space is $A\left(\bar{x}, x_{e}, x_{o}\right) \equiv\left\langle\bar{x}, x_{e}, x_{o} \mid A\right\rangle$ where $o=1,3,5, \ldots$ and $e=2,4,6, \ldots$ denote excited modes and $\bar{x}$ is the midpoint mode. In the mixed even positions and odd momenta space (obtained by Fourier transformation) the probability amplitude is $A\left(\bar{x}, x_{e}, p_{o}\right) \equiv\left\langle\bar{x}, x_{e}, p_{o} \mid A\right\rangle$. As in [1] we define $p_{e}$ as a linear combination of the odd momentum modes $p_{e}=\Sigma_{o} p_{o} R_{o e}$ leading to the probability amplitude $\left\langle\bar{x}, x_{e}, p_{e} \mid A\right\rangle=A\left(\bar{x}, x_{e}, p_{e}\right)$. It is important to emphasize that here $p_{e}$ is not the momentum that is quantum canonical conjugate to $x_{e}$ as defined in the canonical treatment of string modes. That mode is represented by $-i \partial_{x_{e}}$ as applied on the string field $A\left(\bar{x}, x_{e}, p_{e}\right)$. Instead, $p_{e}$ is defined as a linear combination of the odd momentum modes as above. Since $x_{e}$ and $p_{o}$ commute in quantum mechanics, $x_{e}$ and $p_{e}$ also commute with each other in quantum mechanics, and therefore $\left(x_{e}, p_{e}\right)$ are quantum mechanically compatible observables, as they should be in defining the probability amplitude. At first sight it appears somewhat misleading to use the symbol $p_{e}$, since that symbol is also used for the quantum canonical conjugate to $x_{e}$. However, the string joining operation introduces a new noncommutativity beyond quantum mechanics, such that $x_{e}^{\mu}, p_{e}^{\mu}$ behave like a canonical pair under the string joining star product as in Eq. (1.3), although $x_{e}, p_{e}$ commute with each other in quantum mechanics. In this sense the usual momentum mode $-i \partial_{x_{e}} A$ is actually reproduced as a star commutator $-i \partial_{x_{e}} A=\left[p_{e}, A\right]_{\star}$, and therefore, after all, $p_{e}$ does behave as if it is a canonical conjugate to $x_{e}$, justifying the use of the symbol $p_{e}$.
} 
bining their first-quantized probability amplitudes (i.e. string fields) $A_{1}\left(\bar{x}, x_{e}, p_{e}\right)$ and $A_{2}\left(\bar{x}, x_{e}, p_{e}\right)$ into the probability amplitude $A_{12}\left(\bar{x}, x_{e}, p_{e}\right)$

$$
A_{12}\left(\bar{x}, x_{e}, p_{e}\right)=A_{1}\left(\bar{x}, x_{e}, p_{e}\right) \star A_{2}\left(\bar{x}, x_{e}, p_{e}\right),
$$

where the star product is local at the midpoint $\bar{x}^{\mu}$ of both strings, and is precisely the Moyal product in the noncommutative space $x_{e}, p_{e}$, separately for each $e=2,4,6, \ldots$, as in [1]

$$
\left.\star \equiv \prod_{e \geqslant 2} e^{(i / 2)\left(\hat{\partial}_{x_{e}}\right.} \vec{\partial}_{p_{e}}-\bar{\partial}_{p_{e}} \vec{\partial}_{x_{e}}\right) .
$$

This formulation of string interactions, which reproduces the operator formalism or conformal field theory, has developed into an efficient computational tool in string field theory [1-4].

The meaning of the symbol $p_{e}^{\mu}$ must be emphasized. Namely, as described in footnote 2 , it must be clearly understood that the canonical-conjugate-like properties of $p_{e}$ in MSFT

$$
x_{e}^{\mu} \star p_{e^{\prime}}^{\nu}-p_{e^{\prime}}^{\nu} \star x_{e}^{\mu}=i \delta_{e e^{\prime}} \eta^{\mu \nu},
$$

derive from string joining, and not from quantum mechanics. ${ }^{3}$ Thus the process of string joining creates the noncommutative space $\left(x_{e}^{\mu}, p_{e}^{\mu}\right)$ which includes timelike coordinates and is Lorentz covariant. The ghost problems of the timelike coordinates is taken care of by the overall gauge invariance structure of MSFT.

Since the star product above is independent for each string mode, we may concentrate on the supersymmetrization of the Moyal product for one degree of freedom. To do this we are inspired by the close relation between the string joining star product for a single mode as given in Eq. (1.3), and the quantum mechanics of a single relativistic particle which has exactly the same mathematical structure. That is, to supersymmetrize the string joining star product we will borrow from the supersymmetry structure of the quantum mechanics of the relativistic supersymmetric particle.

Thus we consider the phase space degrees of freedom of a single relativistic superparticle given by $\left(x^{\mu}, p^{\mu}, \theta^{\alpha}, \pi_{\alpha}\right)$, where $\mu$ denotes the vector and $\alpha$ denotes the spinor in $d$ dimensions, with $\left(x^{\mu}, p^{\mu}\right)$ and $\left(\theta^{\alpha}, \pi_{\alpha}\right)$ being canonical conjugates. However, there is also a fermionic constraint such that $\pi_{\alpha}$ is not an independent degree of freedom, and results in the supercharge being proportional to $\theta$

$$
Q_{\alpha} \sim(\not \theta \theta)_{\alpha}, \quad(\not p)_{\alpha \beta} \equiv p_{\mu}\left(\gamma^{\mu}\right)_{\alpha \beta} .
$$

Hence we may take only $\left(x^{\mu}, p^{\mu}, Q_{\alpha}\right)$ as the independent degrees of freedom, and consider fields in super phase space of the form $A(x, p, Q)$. We can think of these functions as the

\footnotetext{
${ }^{3}$ However, this observation leads us to speculate that the mysterious origin of quantum mechanics may be related to some deeper physical phenomenon, analogous to string joining.
}

Weyl images of corresponding operators in quantum mechanics, and define a star product among them such that the star product among the Weyl images reproduces the products of the operators in quantum mechanics. The star product thus defined is the generalization of the Moyal product which is automatically invariant under relativistic supersymmetry transformations. We then propose the same mathematical structure as the supersymmetrization of string joining one mode at a time, generalizing the product in Eq. (1.3).

In the present paper we discuss the superparticle and the corresponding star product in its own right. It remains to be examined in the future whether this proposal for supersymmetrizing one mode at a time really reproduces the joining of superstrings.

Thus we will first propose a novel covariant quantization of the off-shell superparticle in Sec. II, and then study the star product in the noncommutative covariant superspace that emerges in Sec. III. In this approach to quantization of the superparticle, we will deviate from the structure of the superparticle in one respect, namely we will not impose the mass shell condition $p^{2}=0$ which also implies the constraint $p Q$ $=0$. These constraints will be relaxed because the string modes which we wish to consider are off shell and do not satisfy these conditions. Then we find that the quantum theory of the off-shell 10-dimensional superparticle is described by a nonlinear realization of the 11-dimensional Poincaré superalgebra. The superspace thus defined is noncommutative, and it becomes the basis for our proposal for the star product in supersymmetric string field theory.

\section{COVARIANT QUANTIZATION OF THE OFF-SHELL SUPERPARTICLE}

The standard generator of supersymmetry acting on unconstrained super phase space is $Q_{\alpha}=\pi_{\alpha}+(\not p \theta)_{\alpha}$. The commutation rules among $Q_{\alpha}$ and other functions of phase space follow from the canonical commutation rules $\left[x_{\mu}, p_{\nu}\right]=i \eta_{\mu \nu}$ and $\left\{\pi_{a}, \theta^{\beta}\right\}=\delta_{\alpha}^{\beta}$. In particular $Q_{\alpha}$ and $p_{\mu}$ satisfy the standard Poincaré superalgebra

$$
\left\{Q_{\alpha}, Q_{\beta}\right\}=2(p)_{\alpha \beta},\left[Q_{\alpha}, p_{\mu}\right]=0,\left[p_{\mu}, p_{\nu}\right]=0 .
$$

The superparticle is defined with a constrained super phase space. In particular, one finds that the following combination of canonical variables vanishes $d_{\alpha} \equiv \pi_{\alpha}-(\not p \theta)_{\alpha}$ $=0$. Therefore, in the subspace $\pi_{\alpha}=(p \theta)_{\alpha}$ the supercharge $Q_{\alpha}$ takes the form $Q_{\alpha} \sim(p \theta)_{\alpha}$. Due to such relations the independent degrees of freedom need to be identified and then the correct commutation rules need to be worked out for the constrained subspace. Whatever these may turn out to be for some chosen independent degrees of freedom, the supersymmetry algebra of Eq. (2.1) must remain unchanged even for the constrained system, because this algebra is a reflection of the supersymmetry of the theory.

In noncovariant quantization, such as in the light cone gauge, there is no problem in identifying and quantizing the 
independent degrees of freedom ${ }^{4}$ of the massless, on shell $p^{2}=0$, superparticle [12]. However, the covariant quantization of the massless superparticle has been a longstanding problem. Despite many attempts in a variety of approaches that provide an answer consistent with light cone quantization, there is still room for discussion $[13,14]$ of what is an economical approach to covariant quantization. This problem has attracted a lot of attention because it is a first step toward the much harder problem of covariant quantization of the superstring in the Green-Schwarz formalism [15].

In our investigation in this paper we will relax the mass shell condition and allow any value for $p^{2}$. This is a desirable step anyway for massless superparticles which are off shell in the presence of interactions. It is also desirable for the application we have in mind in string field theory as explained in the previous section. Since the constraint due to kappa supersymmetry $p Q=0$ is not satisfied off-shell, the fermionic gauge symmetry is no longer effective. Hence the off-shell superparticle has more degrees of freedom. In this case we see that, at least heuristically, we can solve for $\theta$ from the constraint $\theta_{\alpha} \sim\left(\not p^{-1} Q\right)_{\alpha}$, where $\left(\not p^{-1}\right)_{\alpha \beta}$ $=1 / p^{2} p_{\alpha \beta}$, so that we may attempt to formulate the quantum theory covariantly in terms of the off-shell independent degrees of freedom $\left(Q_{\alpha}, p_{\mu}, x_{\mu}\right)$, while treating $\theta$ as a dependent quantity. We preferred to eliminate $\theta$ and keep $Q$ as the independent dynamical quantity since the commutation relations of $Q$ with any quantity have the meaning of infinitesimal supersymmetry transformations and therefore its commutators can be obtained from SUSY transformations. In particular we already know the quantum algebra of $(Q, p)$ through the SUSY algebra in Eq. (2.1). As we have already emphasized, the SUSY algebra must be obeyed in any procedure of quantization because of consistency with the underlying global symmetry of the theory.

What remains is to find the commutation rules of $x^{\mu}$. In particular we need to find $\left[Q_{\alpha}, x^{\mu}\right]$ and $\left[x^{\mu}, x^{\nu}\right]$. These are generally gauge dependent since $x^{\mu}$ transforms under the fermionic local symmetry as well as under the reparametrization symmetry. To find these we will require consistency with the covariant canonical commutation rule

$$
\left[x^{\mu}, p^{\nu}\right]=i \eta^{\mu \nu},
$$

and with the covariant SUSY algebra of Eq. (2.1).

\footnotetext{
${ }^{4}$ In the light cone gauge one chooses $\gamma^{+} \theta=0$ and $x^{+}=p^{+} \tau$, and the mass shell condition $p^{2}=0$ is solved by $p^{-}=\left(p^{i}\right)^{2} /\left(2 p^{+}\right)$. In 10 dimensions, the remaining independent degrees of freedom are $\left(x^{-}, x^{i} ; p^{+}, p^{i}, \chi_{a}\right)$ where $i=1, \ldots, 8$ labels $\mathrm{SO}(8)$ vectors and $a$ $=1, \ldots, 8$ labels $\mathrm{SO}(8)$ spinors. Using the following $16 \times 16$ gamma matrix representation in the light cone basis $\gamma^{+}=\left(\begin{array}{c}\sqrt{2} 0 \\ 00\end{array}\right), \gamma^{-}=\left(\begin{array}{c}00 \\ -\sqrt{2} 0\end{array}\right), \gamma^{i}=\left(\begin{array}{cc}\sigma^{i} & 0 \\ 0-\sigma^{i}\end{array}\right)$, we can write $\not p=-p^{+} \gamma^{-}$ $-p^{-} \gamma^{+}+p^{i} \gamma^{i}$, and obtain the gauge fixed forms $\theta=\sqrt{\sqrt{2} / p^{+}}\left(\begin{array}{l}\chi \\ 0\end{array}\right)$ and $Q=\not p \theta=\sqrt{\sqrt{2} / p^{+}}\left(\begin{array}{c}p^{i} \sigma^{i} \chi \\ \sqrt{2} p^{+} \chi\end{array}\right)$. We can then show that the basic supersymmetry algebra in Eq. (2.1) follows from the commutation rules of the independent canonical variables $\left(x^{-}, x^{i} ; p^{+}, p^{i}, \chi_{a}\right)$ given by $\left[x^{-}, p^{+}\right]=-i,\left[x^{i}, p^{j}\right]=i \delta^{i j},\left\{\chi_{a}, \chi_{b}\right\}$ $=\delta_{a b}$.
}

Since $\left[Q_{\alpha}, x^{\mu}\right]$ amounts to an infinitesimal SUSY transformation, we recall that, for unconstrained superspace it was given as $-i\left(\gamma^{\mu} \theta\right)_{\alpha}$. Using this as a hint, and noting that $\theta$ $\sim\left(p^{-1} Q\right)$, we write $\left[Q_{\alpha}, x^{\mu}\right]=-i c\left(\gamma^{\mu} p^{-1} Q\right)_{\alpha}$ where we determine the unknown constant $c$ by consistency with Jacobi identities. Specifically, the super Jacobi identity for $\left(Q_{\alpha}, Q_{\beta}, x^{\mu}\right)=0$, together with Eqs. (2.1), (2.2) determine $c=1 / 2$. Therefore we find

$$
\left[Q_{\alpha}, x^{\mu}\right]=-\frac{i}{2}\left(\gamma^{\mu} p^{-1} Q\right)_{\alpha}
$$

Next we examine the Jacobi identity for $\left(x^{\mu}, x^{\nu}, Q_{\alpha}\right)=0$ to find the commutator $\left[x^{\mu}, x^{\nu}\right]$. We can easily see that this commutator cannot vanish, and therefore we must have a noncommutative space $x^{\mu}$. Using symmetry properties of gamma matrices in 10 dimensions, Lorentz covariance, and dimensions of operators $\left(\frac{1}{2}, 1,-1\right)$ respectively for $(Q, p, x)$, we can guess the only possible structure to be $\left[x^{\mu}, x^{\nu}\right]$ $=\left[b /\left(p^{2}\right)^{2}\right] Q\left\{\gamma^{\mu \nu}, p\right\} Q$ up to the unknown constant $b$. Imposing the Jacobi identity $\left(x^{\mu}, x^{\nu}, Q_{\alpha}\right)=0$ we find $b$ $=-1 / 16$. Therefore, we have

$$
\left[x^{\mu}, x^{\nu}\right]=-i \frac{S^{\mu \nu}}{p^{2}}, S^{\mu \nu} \equiv \frac{-i}{16 p^{2}} Q\left\{\gamma^{\mu \nu}, p\right\} Q
$$

This noncommutative algebra among the $x^{\mu}$ is consistent with the commutative subspace in the light cone gauge, as seen from $p Q \rightarrow 0$ if one uses the light cone form ${ }^{5}$ of $Q$ and $p$ in footnote 4 .

In preparation for the Jacobi identity among three $x^{\mu}$ s we evaluate

$$
\left[\left[x^{\mu}, x^{\nu}\right], x^{\lambda}\right]=\frac{1}{p^{4}}\left(p^{\mu} S^{\lambda \nu}-p^{\nu} S^{\lambda \mu}+2 p^{\lambda} S^{\mu \nu}\right) .
$$

To arrive at this form we used a number of gamma matrix identities, and the form $Q \gamma^{\mu} Q=p^{\mu}$ which follows from the symmetric $\left(\gamma^{\mu}\right)_{\alpha \beta}$ and the anticommutator in Eq. (2.1). From this, it is immediately seen that the Jacobi identity is satisfied $\left(x^{\mu}, x^{\nu}, x^{\lambda}\right)=0$. All other Jacobi identities among the quantities $Q_{\alpha}, p^{\mu}, x^{\mu}$ are trivially satisfied.

Hence we have shown that the off-shell covariant quantization of the superparticle is uniquely determined by the basic commutations rules in Eqs. (2.1)-(2.4). In our covariant quantization approach we were guided only by the consistency with the global symmetry. The consistent nonlinear algebra defined by these equations is a nonlinear extension of the well known SUSY algebra of Eq. (2.1).

\footnotetext{
${ }^{5}$ Due to powers of $p^{2}$ in the denominator, the on-shell massless particle condition $p^{2}=0$ leads to ambiguous expressions $0 / 0$. However, the light cone massless particle is defined by eliminating some of its degrees of freedom through gauge conditions, and this procedure requires us to interpret $0 / 0$ as zero when comparing to the light cone quantization of the massless particle.
} 
The results above can also be derived from the Dirac brackets in covariant quantization as follows. The constraints $d_{\alpha} \equiv \pi_{\alpha}-(\not \theta)_{\alpha}$ obey the algebra $\left\{d_{\alpha}, d_{\beta}\right\}=-2 p_{\alpha \beta}$. When $p^{\mu}$ is off shell these are second class constraints. Using the constraints $d_{\alpha}$, one can compute the classical Dirac brackets among $\left(x^{\mu}, p_{\mu}, \theta^{\alpha}, \pi_{\alpha}\right)$ as follows:

$$
\begin{aligned}
\left\{x^{\mu}, p_{\nu}\right\}_{D} & =\delta_{\nu}^{\mu}, \quad\left\{x^{\mu}, \theta^{\alpha}\right\}_{D}=-\frac{1}{2}\left(p^{-1} \gamma^{\mu} \theta\right)^{\alpha}, \\
\left\{x^{\mu}, \pi_{\alpha}\right\}_{D} & =\frac{1}{2}\left(\gamma^{\mu} \theta\right)_{\alpha}, \\
\left\{\theta^{\alpha}, \theta^{\beta}\right\}_{D} & =\frac{1}{2}\left(p^{-1}\right)^{\alpha \beta},\left\{\theta^{\alpha}, \pi_{\beta}\right\}_{D}=\frac{1}{2} \delta_{\beta}^{\alpha}, \\
\left\{\pi_{\alpha}, \pi_{\beta}\right\}_{D} & =\frac{1}{2} \not p_{\alpha \beta}, \\
\left\{x^{\mu}, x^{\nu}\right\}_{D} & =\frac{i}{4} \theta\left\{\gamma^{\mu \nu}, p^{-1}\right\} \theta .
\end{aligned}
$$

Solving the constraints one can write $\pi_{\alpha}=(\not p \theta)_{\alpha}, Q_{\alpha}$ $=2 \pi_{\alpha}$ and $\theta_{\alpha}=\frac{1}{2}\left(p^{-1} Q\right)_{\alpha}$. Eliminating $\theta$ and $\pi$ in favor of $Q$ through these equations, and inserting the factor of $i$ in passing to quantum mechanics, we arrive at the same relations derived above through the Jacobi identities.

Next, we examine further the properties of $S^{\mu \nu}$. We see that it commutes with the momentum $p^{\mu}$, it is transverse to it $S^{\mu \nu} p_{\nu}=0$, and from Eq. (2.1) it follows that it satisfies the algebra of Lorentz transformations in the space transverse to $p^{\mu}$

$$
\left[S^{\mu \nu}, S^{\lambda \sigma}\right]=i\left(S^{\mu \lambda}\left(\eta^{\nu \sigma}-\frac{p^{\nu} p^{\sigma}}{p^{2}}\right)-(\mu \leftrightarrow \nu)\right)-i((\lambda \leftrightarrow \sigma)) .
$$

Therefore, $S^{\mu \nu}$ is interpreted as the spin operator. Indeed, its commutator with $Q_{\alpha}$, as follows from Eq. (2.1), gives the correct Lorentz transformation of the spinor in the subspace transverse to $p^{\mu}$

$$
\left[S^{\mu \nu}, Q_{\alpha}\right]=\frac{i}{2}\left(\left(\gamma^{\mu \nu}-\gamma^{\mu \sigma} \frac{p^{\nu} p_{\sigma}}{p^{2}}+\gamma^{\nu \sigma} \frac{p^{\mu} p_{\sigma}}{p^{2}}\right) Q\right)_{\alpha}
$$

These observations lead us to introduce the following dimensionless Hermitian vector $J^{\mu}$

$$
\begin{aligned}
J^{\mu} & \equiv\left(-p^{2}\right)^{1 / 4} x^{\mu}\left(-p^{2}\right)^{1 / 4}=\left(-p^{2}\right)^{1 / 2} x^{\mu}-\frac{i}{2}\left(-p^{2}\right)^{-1 / 2} p^{\mu} \\
& =x^{\mu}\left(-p^{2}\right)^{1 / 2}+\frac{i}{2}\left(-p^{2}\right)^{-1 / 2} p^{\mu}
\end{aligned}
$$

We find that its commutators give the total Lorentz generator $J^{\mu \nu}$

$$
\left[J^{\mu}, J^{\nu}\right]=i J^{\mu \nu}, \quad J^{\mu \nu}=\left(x^{\mu} p^{\nu}-x^{\nu} p^{\mu}\right)+S^{\mu \nu} .
$$

It is now straightforward to notice that the operators $J^{\mu \nu}, p^{\mu}, Q_{\alpha}$ satisfy the super Poincaré algebra. Namely $J_{\mu \nu}$ rotates correctly $p^{\mu}$ as well as $Q_{\alpha}$, and it satisfies the Lorentz algebra not only in the space transverse to $p^{\mu}$, but in the full space

$$
\begin{gathered}
{\left[J^{\mu \nu}, p^{\lambda}\right]=i \eta^{\lambda[\mu} p^{\nu]},\left[J^{\mu \nu}, Q_{\alpha}\right]=\frac{i}{2}\left(\gamma^{\mu \nu} Q\right)_{\alpha}} \\
{\left[J^{\mu \nu}, J^{\lambda \sigma}\right]=i\left(J^{\mu \lambda} \eta^{\nu \sigma}-(\mu \leftrightarrow \nu)\right)-i(\lambda \leftrightarrow \sigma) .}
\end{gathered}
$$

Hence our covariant quantization of the off shell superparticle is consistent with the global Poincare symmetry of the theory.

Furthermore, we can extend the symmetry algebra into a hidden symmetry in 11 dimensions, by including the operators $J^{\mu},\left(-p^{2}\right)^{1 / 2}$, and $\widetilde{Q}_{\alpha} \equiv\left(-p^{2}\right)^{-1 / 2}(p Q)_{\alpha}$. We compute the commutators of $J^{\mu}$ with the other quantities and find

$$
\begin{aligned}
{\left[J^{\mu},\left(-p^{2}\right)^{1 / 2}\right] } & =-i p^{\mu},\left[J^{\mu}, p^{\nu}\right]=i \eta^{\mu \nu}\left(-p^{2}\right)^{1 / 2} \\
{\left[J^{\mu}, J^{\nu \lambda}\right] } & =-i \eta^{\mu[\nu} J^{\lambda]}, \\
{\left[J^{\mu}, Q_{\alpha}\right] } & =-\frac{i}{2}\left(\gamma^{\mu} \widetilde{Q}\right)_{\alpha},\left[J^{\mu}, \widetilde{Q}_{\alpha}\right]=\frac{i}{2}\left(\gamma^{\mu} Q\right)_{\alpha}
\end{aligned}
$$

Together with Eqs. (2.13), (2.14), we notice the structure of the 11-dimensional SUSY algebra, such that $Q_{A}=\left(Q_{\alpha}, \widetilde{Q}_{\dot{\alpha}}\right)$ together form a 32 component spinor, $P^{M}=\left(p^{\mu},\left(-p^{2}\right)^{1 / 2}\right)$ together form an 11-dimensional massless momentum, and $J^{M N}=\left(J_{\mu \nu}, J_{\mu}\right)$ together form the 11-dimensional Lorentz algebra. Furthermore, if we define 11-dimensional gamma matrices $\Gamma^{M}$, we can check explicitly that $P^{M}\left(\Gamma_{M}\right)_{A}^{B} Q_{B}$ $=0$ as well as $P^{M} P_{M}=0$, therefore our structure corresponds to the quantum massless superparticle in 11 dimensions. The quantum states of this off-shell system are precisely the supergravity multiplet in 11 dimensions, but dimensionally reduced to the 10-dimensional type-IIA supergravity multiplet.

Thus our covariant quantization of the off-shell 10 dimensional superparticle is described by a nonlinear realization of the 11-dimensional super Poincaré algebra, acting on the quantum states that correspond to the 11-dimensional supergravity multiplet. The fundamental commutators of this structure are given by Eqs. (2.1)-(2.4), including the noncommutative position space of Eq. (2.4). In particular the commutation rules with the operator $Q_{\alpha}$ correspond to the supersymmetry transformations of the fundamental supercoordinates $\left(x^{\mu}, p^{\mu}, Q_{\alpha}\right)$, and this gives the supersymmetry transformation rules of fields defined as functions of this superspace $A(x, p, Q)$.

In our discussion we treated the 10-dimensional superparticle, but it is straightforward to apply the same approach in any dimension $d$, leading to a nonlinear realization of the Poincaré superalgebra in $d+1$ dimensions. 
The nonlinear superalgebra among the noncommutative covariant superspace coordinates $\left(x^{\mu}, p^{\mu}, Q_{\alpha}\right)$ is the basis for constructing the supersymmetric star product. In principle one can use the Kontsevich method $[16,17]$ to construct the associative star product. However, we can use simpler methods that can be applied to our problem as developed in the next section. Thus, we will first discuss a generic linear case and later apply the method to the nonlinear superalgebra in our case.

\section{STAR PRODUCT IN NONCOMMUTATIVE SUPERSPACE}

\section{A. At most linear Poisson structure}

Consider some noncommutative space $x_{a}$ with a noncommutativity function $\theta_{a b}(x)$, such that $\theta_{a b}(x)$ is at most linear in $x$. The classical Poisson structure then has the form

$$
\left\{x_{a}, x_{b}\right\}=-i \theta_{a b}(x)=-i\left(\sigma_{a b}+i f_{a b}^{c} x_{c}\right),
$$

where $\sigma_{a b}, f_{a b}^{c}$ are independent of $x$. Such a $\theta_{a b}(x)$ includes the case of the Heisenberg algebra (when $f_{a b}^{c}=0$ ) as well as the case of a pure Lie algebra (when $\sigma_{a b}=0$ ). In the next section we will study the case of superspace with the $x_{a}$ replaced by the phase space of a superparticle $x_{a}$ $\rightarrow\left(x_{\mu}, p_{\mu}, Q_{\alpha}\right)$ where $Q_{\alpha}$ is the generator of supersymmetry. But in the present section we discuss the star product for the general bosonic system that has the general noncommutativity property given above.

The usual quantization of this system is done in quantum mechanics by promoting the $x_{a}$ to operators $\hat{x}_{a}$. General operators $\hat{A}_{1}(\hat{x}), \hat{A}_{2}(\hat{x})$ are multiplied with each other in the usual way by writing them next to each other $\hat{A}_{12}(\hat{x})$ $=\hat{A}_{1}(\hat{x}) \hat{A}_{2}(\hat{x})$, and then the resulting operator $\hat{A}_{12}(\hat{x})$ is computed by keeping track of the orders of the operators consistently with their quantum commutation relations

$$
\left[\hat{x}_{a}, \hat{x}_{b}\right]=\theta_{a b}(\hat{x})=\sigma_{a b}+i f_{a b}^{c} \hat{x}_{c} .
$$

The deformation quantization of this system introduces a star product among classical functions $A_{1}(x), A_{2}(x)$ to construct a resulting classical function $A_{12}(x)$

$$
A_{12}(x)=A_{1}(x) \star A_{2}(x) .
$$

The classical $A_{1}(x), A_{2}(x), A_{12}(x)$ are "Weyl images" of the corresponding operators $\hat{A}_{1}(\hat{x}), \hat{A}_{2}(\hat{x}), \hat{A}_{12}(\hat{x})$ and are designed to reproduce the same results as quantum mechanics, although in the classical function $A(x)$ the orders of $x_{a}$ do not matter. In particular, when applied to the case of $A_{1}(x)$ $=x_{a}, A_{2}(x)=x_{b}$, the star product should give the same result as quantum mechanics

$$
\left[x_{a}, x_{b}\right]_{\star} \equiv x_{a} \star x_{b}-x_{b} \star x_{a}=\theta_{a b}(x)=\sigma_{a b}+i f_{a b}^{c} x_{c} .
$$

An additional property of the star product, consistent with quantum mechanics, is that it should be associative

$$
A_{123}(x)=\left(A_{1}(x) \star A_{2}(x)\right) \star A_{3}(x)=A_{1}(x) \star\left(A_{2}(x) \star A_{3}(x)\right) .
$$

A star product with such properties can be constructed by using Kontsevich's general diagrammatic prescription [16], which defines it as an infinite series for an arbitrary $\theta_{a b}(x)$. However, because of the maximum linear nature of $\theta_{a b}(x)$ there is a much simpler closed expression which we construct in the following section. Of course, we expect that in the limit $f_{a b}^{c}=0$, our expression reduces to the simple Moyal product given by $\theta_{a b} \rightarrow \sigma_{a b}$.

\section{B. The generic star for maximum linear $\theta_{a b}(x)$}

We define the Fourier transforms of the classical functions

$$
A_{i}(x)=\int(d p) \tilde{A}_{i}(p) e^{i p^{a} x_{a}} .
$$

Constructing the star product for the Fourier basis $e^{i p_{1}^{a} x_{a}} e^{i p_{2}^{b} x_{b}}$ is equivalent to constructing it for any other basis of functions that can be related by the Fourier transform. We recall the Baker-Hausdorff-Campbell (BHC) theorem for quantum operators

$$
e^{\hat{A}} e^{\hat{B}}=e^{\hat{C}(\hat{A}, \hat{B})},
$$

where the operator $\hat{C}(\hat{A}, \hat{B})$ is determined by an infinite series given by multiple commutators

$$
\begin{aligned}
\hat{C}(\hat{A}, \hat{B})= & \hat{A}+\hat{B}+\frac{1}{2}[\hat{A}, \hat{B}]+\frac{1}{12}[[\hat{A}, \hat{B}], \hat{B}] \\
& +\frac{1}{12}[\hat{A},[\hat{A}, \hat{B}]]+\cdots
\end{aligned}
$$

We note that this theorem relies simply on an associative product. Therefore, the same theorem also applies to star exponentials of classical functions $A(x), B(x)$ as long as we have an associative star product

$$
\left(e^{A(x)}\right)_{\star} \star\left(e^{B(x)}\right)_{\star}=\left(e^{\left(A(x)+B(x)+(1 / 2)[A(x), B(x)]_{\star}+(1 / 12)\left[[A, B]_{\star}, B\right]_{\star}+(1 / 12)\left[A,[A, B]_{\star}\right]_{\star}+\cdots\right)}\right)_{\star} .
$$


In particular, let us construct a star product such that the star exponential of a linear function of the $x^{a}$ is equal to the classical exponential, i.e. $\left(e^{i p \cdot x}\right)_{\star}=e^{i p \cdot x}$ for any set of constant parameters $p^{a}$. This relation is true for the simple Moyal product, and we will verify that it is also true in our case, after we give the construction of our star product. Then the BHC theorem can be applied to the classical Fourier basis

$$
\begin{aligned}
e^{i p p_{1}^{a} x_{a}} \star e^{i p_{2}^{b} x_{b}}= & \exp \left(i p_{1}^{a} x_{a}+i p_{2}^{a} x_{a}\right. \\
& \left.+\frac{1}{2}\left[i p_{1}^{a} x_{a}, i p_{2}^{b} x_{b}\right]_{\star}+\cdots\right) .
\end{aligned}
$$

The crucial observation here is that all the higher terms in $C(A, B)(x)$ involve only star commutators, which can be evaluated for the Fourier basis by using repeatedly Eq. (3.4). Since $\theta_{a b}(x)$ is at most linear in $x$, the result is also necessarily at most linear in $x$, and therefore only the linear Poisson structure is sufficient to completely evaluate the product of exponentials. The result is identified with the classical exponential since it is designed to be the same as the star exponential for any $p$. Therefore, we obtain the form

$$
e^{i p_{1}^{a} x_{a} \star e^{i p_{2}^{a} x_{a}}}=Z\left(p_{1}, p_{2}\right) \exp \left(i x_{a} p_{12}^{a}\right),
$$

where $Z, p_{12}^{a}$ are functions of $p_{1}^{a}, p_{2}^{a}$ which we determine below. Once these functions are determined, the star product for generic classical functions $A_{1}(x), A_{2}(x)$ can be given exactly in the form

$$
\begin{aligned}
A_{12}(x)= & A_{1}(x) \star A_{2}(x) \\
= & \iint\left(d p_{1} d p_{2}\right) \widetilde{A}_{1}\left(p_{1}\right) \widetilde{A}_{2}\left(p_{2}\right) Z\left(p_{1}, p_{2}\right) \\
& \times \exp \left(i x_{a} p^{a}\left(p_{1}, p_{2}\right)\right) .
\end{aligned}
$$

This can be rewritten in terms of differentials as follows

$$
\begin{aligned}
A_{1}(x) \star A_{2}(x)= & {\left[A_{1}\left(x_{1}\right) Z\left(-i \overleftarrow{\partial}_{x_{1}},-i \vec{\partial}_{x_{2}}\right)\right.} \\
& \times \exp \left(i x_{a} \Delta p^{a}\left(-i \overleftarrow{\partial}_{x_{1}},-i \vec{\partial}_{x_{2}}\right)\right) \\
& \left.\times\left(A_{2}\left(x_{2}\right)\right)\right]_{x_{1}=x_{2}=x}
\end{aligned}
$$

where $\Delta p^{a}\left(p_{1}, p_{2}\right)=p_{12}^{a}\left(p_{1}, p_{2}\right)-p_{1}^{a}-p_{2}^{a}$. Note that $\Delta p^{a}$ is multiplied by $x^{a}$ which is kept distinct from $x_{1}^{a}, x_{2}^{a}$ when the derivatives are applied. The derivative form is useful in general, but it is particularly essential for evaluating the star products of polynomials in $x^{a}$.

In the simplest case of $f_{a b}^{c}=0$ the infinite series in Eq. (3.10) terminates with the first commutator $\frac{1}{2}\left[i p_{1}^{a} x_{a}, i p_{2}^{a} x_{a}\right]_{\star}=-\frac{1}{2} p_{1}^{a} \sigma_{a b} p_{2}^{b}$ since it is independent of $x$. Similarly, for certain cases of interest (for an example see [18]), the series terminates after a few terms once we reach terms that are independent of $x$. Even if the series does not terminate, the functions $Z\left(p_{1}, p_{2}\right), p_{12}^{a}\left(p_{1}, p_{2}\right)$ can be com- puted by using the properties of the Lie algebra associated with the structure constants $f_{a b}^{c}$.

In particular, when we specialize this expression to $f_{a b}^{c}$ $=0$, we have $Z\left(p_{1}, p_{2}\right)=\exp \left(-\frac{1}{2} p_{1}^{a} \sigma_{a b} p_{2}^{b}\right)$ and $p_{12}^{a}\left(p_{1}, p_{2}\right)$ $=p_{1}^{a}+p_{2}^{a}$, which gives

$$
\begin{aligned}
A_{12}(x)= & A_{1}(x) \star A_{2}(x) \underset{f_{a b}^{c}=0}{=} \iint\left(d p_{1} d p_{2}\right) \\
& \times \widetilde{A}_{1}\left(p_{1}\right) \widetilde{A}_{2}\left(p_{2}\right) e^{-(1 / 2) p_{1}^{a} \sigma_{a b} p_{2}^{b}} e^{i x_{a}\left(p_{1}^{a}+p_{2}^{a}\right)} .
\end{aligned}
$$

The right hand side may be written also in the derivative form $A_{1}(x) \star A_{2}(x)=A_{1}(x) \exp \left(\frac{1}{2} \bar{\partial}^{a} \sigma_{a b} \vec{\partial}^{b}\right) A_{2}(x)$, which shows that the result is the usual Moyal star product when $f_{a b}^{c}$ $=0$.

For the general case, we compute the first few terms of the series for any $\sigma_{a b}, f_{a b}^{c}$

$$
\begin{aligned}
\frac{1}{2}\left[i p_{1}^{a} x_{a}, i p_{2}^{b} x_{b}\right]_{\star}= & -\frac{1}{2} p_{1}^{a} \sigma_{a b} p_{2}^{b}-\frac{i}{2}\left(p_{1}^{a} p_{2}^{b} f_{a b}^{c}\right) x_{c} \\
\frac{1}{12}\left[\left[i p_{1}^{a} x_{a}, i p_{2}^{b} x_{b}\right], i p_{2}^{c} x_{c}\right]= & \frac{1}{24}\left(p_{1}^{a} f_{a b}^{d} p_{2}^{b}\right) \sigma_{d c} p_{2}^{c} \\
& +\frac{i}{24}\left(p_{1}^{a} p_{2}^{b} p_{2}^{c} f_{a b}^{d} f_{d c}^{e}\right) x_{e} .
\end{aligned}
$$

So we obtain

$$
\begin{aligned}
Z\left(p_{1}, p_{2}\right)= & \exp \left(-\frac{1}{2} p_{1}^{a} \sigma_{a b} p_{2}^{b}+\frac{1}{24}\left(p_{1}^{a} f_{a b}^{d} p_{2}^{b}\right) \sigma_{d c} p_{2}^{c}\right. \\
& \left.+\frac{1}{24}\left(p_{2}^{a} f_{a b}^{d} p_{1}^{b}\right) \sigma_{d c} p_{1}^{c}+\cdots\right) \\
p_{12}^{e}\left(p_{1}, p_{2}\right)= & p_{1}^{e}+p_{2}^{e}-\frac{1}{2} p_{1}^{a} f_{a b}^{e} p_{2}^{b}+\frac{1}{24}\left(p_{1}^{a} f_{a b}^{d} p_{2}^{b}\right) f_{d c}^{e} p_{2}^{c} \\
& +\frac{1}{24}\left(p_{2}^{a} f_{a b}^{d} p_{1}^{b}\right) f_{d c}^{e} p_{1}^{c}+\cdots .
\end{aligned}
$$

We identify the structure of the series as follows. First, $p_{12}^{e}\left(p_{1}, p_{2}\right)$ is independent of $\sigma_{a b}$; it is fully determined by the group multiplication property, with $p_{1}^{a}, p_{2}^{b}$ being the infinitesimal group parameters associated with the Lie algebra characterized by $f_{a b}^{c}$. Therefore, the full series for $p_{12}^{e}\left(p_{1}, p_{2}\right)$ can be computed from any convenient representation of the group (see below). Second, the expression for $\ln Z\left(p_{1}, p_{2}\right)$ is completely parallel to the expression for $p_{12}^{e}\left(p_{1}, p_{2}\right)-p_{1}^{e}-p_{2}^{e}$, except for replacing $\sigma_{a b}$ in place of $f_{a b}^{e}$ in the last factor of each term. In fact, $\sigma_{a b}$ may be regarded as an additional structure constant in the centrally extended Lie algebra characterized by $f_{a b}^{e}$, which explains why the two series for $\ln Z\left(p_{1}, p_{2}\right)$ and $p_{12}^{e}\left(p_{1}, p_{2}\right)$ have a similar structure. Thus, if $p^{e}\left(p_{1}, p_{2}\right)$ is computed exactly from some convenient group representation, $\ln Z\left(p_{1}, p_{2}\right)$ can 
also be computed by using its relationship to $p_{12}^{e}\left(p_{1}, p_{2}\right)$ or by using a convenient representation of the centrally extended Lie algebra.

As an example, consider the case of $\sigma_{a b}=0$ and take $f_{a b}^{c}$ to be the structure constants for $\mathrm{SU}(2)$. To compute $p_{12}^{a}\left(p_{1}, p_{2}\right)$ exactly we use the $2 \times 2$ matrix representation $e^{i x_{a} p^{a}} \rightarrow e^{i(1 / 2) \sigma_{a} p^{a}}$ where $\sigma_{a}$ are the Pauli matrices. Then the matrix representation $e^{i(1 / 2) \sigma_{a} p^{a}}=\cos (|p| / 2)$ $+i(p \cdot \sigma /|p|) \sin (|p| / 2)$ can be used to multiply the matrices and compute an exact expression for $p_{12}^{a}\left(p_{1}, p_{2}\right)$ written as a 3-vector $\vec{p}$ as follows:

$$
\begin{gathered}
\cos \frac{|p|}{2}=\cos \frac{\left|p_{1}\right|}{2} \cos \frac{\left|p_{2}\right|}{2}-\frac{\vec{p}_{1} \cdot \vec{p}_{2}}{\left|p_{1}\right|\left|p_{2}\right|} \sin \frac{\left|p_{1}\right|}{2} \sin \frac{\left|p_{2}\right|}{2} \\
\frac{\vec{p}}{|p|} \sin \frac{|p|}{2}=\left(\begin{array}{c}
\frac{\vec{p}_{1}}{\left|p_{1}\right|} \sin \frac{\left|p_{1}\right|}{2} \cos \frac{\left|p_{2}\right|}{2}+\frac{\vec{p}_{2}}{\left|p_{2}\right|} \sin \frac{\left|p_{2}\right|}{2} \cos \frac{\left|p_{1}\right|}{2} \\
-\frac{\vec{p}_{1} \times \vec{p}_{2}}{\left|p_{1}\right|\left|p_{2}\right|} \sin \frac{\left|p_{1}\right|}{2} \sin \frac{\left|p_{2}\right|}{2}
\end{array}\right) .
\end{gathered}
$$

The first equation gives the length of the vector $|p|$, and after inserting it in the second equation we get the full $\vec{p}$. The expansion of this exact expression in powers of $\vec{p}_{1}, \vec{p}_{2}$ reproduces the infinite series computed through the BHC theorem. Replacing this result for $p_{12}^{a}\left(p_{1}, p_{2}\right)$ in Eq. (3.12) and taking $Z\left(p_{1}, p_{2}\right)=1$ gives the star product for the case of $\sigma_{a b}=0$ and $f_{a b}^{c}$ the $\mathrm{SU}(2)$ structure constants. It is a nontrivial exercise to obtain this result in the diagramatic approach of Kontsevich.

Finally, let us verify that the star exponential is the classical exponential $\left(e^{i p \cdot x}\right)_{\star}=e^{i p \cdot x}$ which was assumed in our approach. This would follow by showing that the star powers are the same as the classical powers $(p \cdot x)_{\star}^{n}=(p \cdot x)^{n}$ for any set of parameters $p^{a}$. This has to be true since the dot product $p \cdot x$ amounts to picking up a single component of $x$ in the direction of $p^{a}$, and for a single component the star product is trivial with itself since the commutator vanishes $[p \cdot x$, $p \cdot x]_{\star}=0$. In any case one can also verify that it is true explicitly by applying the star product of Eq. (3.13) to $(p \cdot x)^{n_{1}} \star(p \cdot x)^{n_{2}}$ to show that it gives $(p \cdot x)^{n_{1}+n_{2}}$. This can be proven by iteration by starting with $n_{1}=n_{2}=1$; and this case is easily computed by using the expansion of the general formulas in Eqs. (3.17), (3.18).

The lesson learned in this section is that we can use the BHC theorem and group theory to determine the star product. The case treated in this section involved a linear Poisson structure. In the next section we will treat a nonlinear (super)Poisson structure, but the essential tool will be again the group theoretical aspect we emphasized in this section. We will use this concept to determine the star product in relativistic noncommutative superspace $\left(x^{\mu}, p^{\mu}, Q_{\alpha}\right)$. From the context we will see that our approach is a more general technique than the particular example, and therefore it can be applied more generally to other bosonic or supersymmetric cases.

\section{Covariant superstar}

We will find it convenient to work with a basis of functions of $(J, p, Q)$ instead of functions of the noncommutative phase space $(x, p, Q)$, and define the star product in the space of $(J, p, Q)$. This is completely general since these are related by a change of variables ${ }^{6} x^{\mu}=J^{\mu}\left(-p^{2}\right)^{-1 / 2}$. The virtue of the basis $(J, p, Q)$ is that these variables have simple commutation rules as quantum operators (i.e. they are part of the Lie algebra of $(d+1)$-dimensional super Poincaré group), and this makes them convenient for the formalism of defining a star product on classical functions of this space. If one desires, the star product in the space of $(x, p, Q)$ can be extracted from the one we define. To emphasize that the star product is defined in the $(J, p, Q)$ space we will denote it with the symbol $\diamond$, while reserving the symbol $\star$ for the $(x, p, Q)$ space.

We begin with the Fourier transform as in Eq. (3.6)

$$
A(J, p, Q)=\int d k d q d \psi \widetilde{A}(k, q, \psi) e^{i k \cdot J+i q \cdot p+i \psi \cdot Q} .
$$

Then we need to evaluate the star product of the Fourier basis $e^{i k_{1} \cdot J+i q_{1} \cdot p+i \psi_{1} \cdot Q} \diamond e^{i k_{2} \cdot J+i q_{2} \cdot p+i \psi_{2} \cdot Q}$. Note that these equations involve conveniently a classical exponential. Fortunately, the classical exponential of an arbitrary linear combination of $(J, p, Q)$ is equal to the star exponential as long as the star product $\diamond$ is given in the $(J, p, Q)$ basis, $\left(e^{i k \cdot J+i q \cdot p+i \psi \cdot Q}\right)_{\diamond}=e^{i k \cdot J+i q \cdot p+i \psi \cdot Q}$, for any set of constants $\left(k_{\mu}, q_{\mu}, \psi_{\alpha}\right)$. This is by virtue of the fact that, as in the previous section, under the $\diamond$ product $(J, p, Q)$ act as generators of the Lie algebra of the $(d+1)$-dimensional super Poincaré group.

We may begin to apply the BHC theorem, as in Eq. (3.9), to evaluate this product. It becomes quickly evident that the series does not terminate since the non-Abelian $J^{\mu \nu}$ is produced in the commutator $\left[J^{\mu}, J^{\nu}\right]_{\diamond}$. However, the product can be determined from group theory since the exponential of any linear combination of generators defines an element of the super Poincare group in $(d+1)$ dimensions. Therefore, the result of the BHC series must accumulate to become the series one obtains in group multiplication, and therefore it must take the form of a general group element on the right hand side

\footnotetext{
${ }^{6}$ To be more careful, the change of variables needs to be consistent with the corresponding quantum operators, and therefore it should involve star multiplication of various factors. However, note that, as can be expected, for a single power of $x^{\mu}$ or $J^{\mu}$ the star product form is equal to its classical form $J^{\mu}=\left(-p^{2}\right)^{1 / 4} \star x^{\mu} \star\left(-p^{2}\right)^{1 / 4}$ $=x^{\mu}\left(-p^{2}\right)^{1 / 2}, \quad$ or $\quad x^{\mu}=\left(-p^{2}\right)^{-1 / 4} \diamond J^{\mu} \diamond\left(-p^{2}\right)^{-1 / 4}$ $=J^{\mu}\left(-p^{2}\right)^{-1 / 2}$, without any corrections of the deformation parameter (which is set equal to 1 in our formalism).
} 


$$
\begin{aligned}
& e^{i k_{1} \cdot J+i q_{1} \cdot p+i \psi_{1} \cdot Q} \diamond e^{i k_{2} \cdot J+i q_{2} \cdot p+i \psi_{2} \cdot Q} \\
& =\left(e^{i\left(k_{12} \cdot J+\omega_{12}^{\mu \nu} J_{\mu \nu}\right)+i\left(q_{12} \cdot p+z_{12} \sqrt{-p^{2}}\right)+i\left(\psi_{12} \cdot Q+\xi_{12} \cdot \tilde{Q}\right)}\right) \diamond .
\end{aligned}
$$

The important point here is that the coefficients $k_{12}^{\mu}, \omega_{12}^{\mu \nu}$, $q_{12}^{\mu}, z_{12},\left(\psi_{12}\right)_{\alpha},\left(\xi_{12}\right)_{\alpha}$ that appear in the exponent must be constant coefficients (independent of $x, p, Q)$. They are functions of $\left(k_{1}, k_{2} ; q_{1}, q_{2} ; \psi_{1}, \psi_{2}\right)$ which can be determined from any convenient representation of the super Poincaré group in $d+1$ dimensions as in the example of Eqs. (3.19), (3.20). In the result we may then replace the nonlinear classical expressions for $J_{\mu \nu}, \widetilde{Q}$,

$$
J^{\mu \nu}=\frac{J^{[\mu} p^{\nu]}}{\sqrt{-p^{2}}}+\frac{i Q\left\{\gamma^{\mu \nu}, \not p\right\} Q}{16\left(-p^{2}\right)}, \quad \widetilde{Q}_{\dot{\alpha}} \equiv\left(-p^{2}\right)^{-1 / 2}(\not p Q)_{\dot{\alpha}}
$$

and $\sqrt{-p^{2}}$, thus obtaining the desired star product for the basis $e^{i k_{1} \cdot J+i q_{1} \cdot p+i \psi_{1} \cdot Q}$.

Note that, because of the nonlinear nature of $J_{\mu \nu}, \widetilde{Q}, \sqrt{-p^{2}}$ as functions of $(J, p, Q)$ the resulting exponential must be a $\diamond$-star exponential. This is understood as follows. A priori we have started with the classical $(J, p, Q)$ as the Weyl images of the quantum operators $(\hat{J}, \hat{p}, \hat{Q})$. Any function of these operators has an image that is computed by replacing each $(\hat{J}, \hat{p}, \hat{Q})$ by its classical image $(J, p, Q)$ but multiplied with each other by using the $\diamond$ product. With that definition, the $J^{\mu \nu}$ and $\widetilde{Q}_{\alpha}$ that appear in the exponent in Eq. (3.22) are constructed by inserting the $\diamond$ product, such as $J^{\mu \nu}=\left(-p^{2}\right)^{-1 / 4} \diamond J^{[\mu} \diamond p^{\nu]} \diamond\left(-p^{2}\right)^{-1 / 4}+\cdots$. Furthermore, when these are multiplied to build the exponential series, one should always use the star product. Then Eq. (3.22) is understood as the image of its corresponding operator equation.

However, there are some simplifications that permit us to substitute the classical forms of $J_{\mu \nu}, \widetilde{Q}, \sqrt{-p^{2}}$ as mentioned above. First, by noting that $\widetilde{Q}_{\alpha}$ and $S_{\mu \nu}$ are constructed only from (anti)commuting operators we realize that they cannot have any corrections from the deformation parameter ${ }^{7} \hbar$ since one can freely change the orders of $(J, p, Q)$ as quantum operators in these expressions (thanks to the symmetry structure of the gamma matrices in $S_{\mu \nu}$ the nontrivial anticommutator between two $Q$ 's does not contribute). Second, since the expression $\left(-p^{2}\right)^{-1 / 4} \diamond J^{[\mu} \diamond p^{\nu]} \diamond\left(-p^{2}\right)^{-1 / 4}$ is Hermitian, its Weyl image must be real. Therefore it cannot have a contribution at first order in $\hbar$ (odd orders are imaginary). It cannot have $\hbar$ corrections to higher orders either because the star product at higher orders involves higher order derivatives that vanish on a function that is linear in $J$ (analogous to the differential operator version of Moyal star).

\footnotetext{
${ }^{7}$ In this paper for convenience we will refer to the deformation parameter as $\hbar$. However if we apply the formalism to describe string joining in string field theory, the deformation parameter is unrelated to the $\hbar$ in quantum mechanics.
}

Hence, the images for the nonlinear expressions for $J_{\mu \nu}, \widetilde{Q}, \sqrt{-p^{2}}$ are simply their classical expressions.

A systematic expansion of the $\diamond$ product in powers of $\hbar$ can be given as follows. The parameter $\hbar$ comes from two sources: first the star exponentiation, and second the coefficients $\left(k_{12}^{\mu}, \ldots\right)$ which we can compute group theoretically to all orders of $\hbar$. Up to second order in $\hbar$ only the expansion of the coefficients $\left(k_{12}^{\mu}, \ldots\right)$ contribute. Starting with the third order the star exponential also contributes. To compute the contribution from the star exponential, one can use the known form of the star product at one lower order. In this way one can obtain systematically a completely explicit form of the $\diamond$ product to all orders of $\hbar$. By applying this method we have verified a posteriori that indeed the expressions for $J_{\mu \nu}, \widetilde{Q}, \sqrt{-p^{2}}$ do not receive any contributions from $\hbar$. Similarly, the statements in footnote 6 can be verified a posteriori.

The result for the $\diamond$ product may also be written in the differential form of Eq. (3.13) (with $\partial / \partial J$ derivatives, not $\partial / \partial x$ derivatives). This last form is appropriate for computing the star product for polynomials ( other functions of $A(x, p, Q)$ after writing them in terms of $(J, p, Q)$. For example, from the group theoretical result in the $J$ basis, we can also determine the superstar product in the $x$ basis $e^{i k_{1} \cdot x+i q_{1} \cdot p+i \psi_{1} \cdot Q}$ by writing it in the $J$ basis as $\left(e^{i k_{1} \cdot J\left(-p^{2}\right)^{-1 / 2}+i q_{1} \cdot p+i \psi_{1} \cdot Q}\right)_{\diamond}$, with a $\diamond$-star exponential.

In the purely bosonic case, the classical basis $e^{i k_{1} \cdot x+i q_{1} \cdot p}$ is simpler than the basis $\left(\exp \left(i k_{1} \cdot x \sqrt{-p^{2}}+i q_{1} \cdot p\right)\right)_{\star}$ $=e^{i k_{1} \cdot J+i q_{1} \cdot p}$, where $\star$ reduces to the standard Moyal product in the absence of fermions. Similarly, the $\diamond$ products for the Fourier basis $e^{i k_{1} \cdot x+i q_{1} \cdot p}$, when expressed in the $(J, p)$ space, should give the same result as the Moyal product (using the Moyal $\star$ )

$$
\begin{aligned}
& e^{i k_{1} \cdot x+i q_{1} \cdot p} \star e^{i k_{2} \cdot x+i q_{2} \cdot p} \\
& \quad=e^{i\left(k_{1}+k_{2}\right) \cdot x+i\left(q_{1}+q_{2}\right) \cdot p} e^{-(i / 2)\left(k_{1} \cdot q_{2}-k_{2} \cdot q_{1}\right)} .
\end{aligned}
$$

Therefore, the following computation is a test of our formalism

$$
\begin{aligned}
& \left(e^{i k_{1} \cdot J\left(-p^{2}\right)^{-1 / 2}+i q_{1} \cdot p}\right)_{\diamond} \diamond\left(e^{i k_{2} \cdot J\left(-p^{2}\right)^{-1 / 2}+i q_{2} \cdot p}\right)_{\diamond} \\
& \quad=\left(e^{i\left(k_{1}+k_{2}\right) \cdot J\left(-p^{2}\right)^{-1 / 2}+i\left(q_{1}+q_{2}\right) \cdot p}\right) \diamond e^{-(i / 2)\left(k_{1} \cdot q_{2}-k_{2} \cdot q_{1}\right)},
\end{aligned}
$$

where only $\diamond$ exponentials must appear. Indeed this is correct. The technical details of this computation will be given in another paper [19].

Thus, as expected, the Moyal product $\star$ in the $(x, p)$ basis and our group theoretical $\diamond$ product in the $(J, p)$ basis are equivalent when the fermions are absent. In the supersymmetric case it remains to be seen whether one basis is superior to the other in practical computations (explicit computations are in progress).

By following this program we can compute the superstar product for general fields 


$$
A(J, p, Q) \star B(J, p, Q) \text { or } A^{\prime}(x, p, Q) \star B^{\prime}(x, p, Q) \text {. }
$$

The result appears complicated but it has a completely tractable group theoretical structure. We hope to give explicit calculations using these formulas in the near future. Our results as well as methods are likely to be useful in various applications. In particular, we hope that it can be used in the formulation of superstring field theory, which was the motivating factor of our investigation.

In this paper we achieved our main goal of formulating the superstar, but along the way we also obtained two other new results. First we gave the quantization of the off shell superparticle in $d$ dimensions and showed that its quantum mechanics gives a nonlinear realization of $(d+1)$-dimensional Poincaré superalgebra. This higher structure was essential for constructing the superstar. Second, we introduced efficient group theoretical methods for constructing and computing star products. We showed that for the maximum linear Poisson structure, as well as for nonlinear Poisson structures that can be embedded as part of nonlinearly realized Lie (super)algebras, we can obtain the exact full (super)star product by using group theory representations. This concept is useful for performing explicit computations involving the superstar in its applications.

\section{ACKNOWLEDGMENTS}

I.B. would like to thank M. Lledo for discussions. I.B. is in part supported by a DOE grant DE-FG03-84ER40168. He is grateful to the Physics Department at UC Berkeley, the Feza Gürsey Institute, and the CERN TH-division for hospitality while this work was performed. C.D. is supported in part by the Turkish Academy of Sciences in the framework of the Young Scientist Program (CD/TÜBA-GEBIP/2002$1-7)$. A.P. and B.Z. are supported in part by the DOE under contract DE-AC03-76SF00098 and in part by the NSF under grant 22386-13067.
[1] I. Bars, Phys. Lett. B 517, 436 (2001); "MSFT: Moyal star formulation of string field theory," hep-th/0211238.

[2] I. Bars and Y. Matsuo, Phys. Rev. D 65, 126006 (2002); 66, 066003 (2002).

[3] I. Bars, I. Kishimoto, and Y. Matsuo, Phys. Rev. D 67, 066002 (2003); 67, 126007 (2003); J. High Energy Phys. 07, 027 (2003).

[4] M.R. Douglas, H. Liu, G. Moore, and B. Zwiebach, J. High Energy Phys. 04, 022 (2002); D.M. Belov and A. Konechny, ibid. 10, 049 (2002); Phys. Lett. B 558, 111 (2003); E. Fuchs, M. Kroyter, and A. Marcus, J. High Energy Phys. 11, 046 (2002); T.G. Erler, “Moyal formulation of Witten's star product in the fermionic ghost sector," hep-th/0205107; "A fresh look at midpoint singularities in the algebra of string fields," hep-th/0304044.

[5] I. Bars and S. MacDowell, Phys. Lett. 71B, 111 (1977).

[6] J. Schwarz and P. van Nieuwenhuizen, Lett. Nuovo Cimento Soc. Ital. Fis. 34, 21 (1982).

[7] J. de Boer, P.A. Grassi, and P. van Nieuwenhuizen, "Noncommutative Superspace From String Theory," hep-th/0302078.
[8] H. Ooguri and C. Vafa, "The C-deformation of gluino and non-planar diagrams," hep-th/0302109; "Gravity induced C-deformation," hep-th/0303063.

[9] N. Seiberg, J. High Energy Phys. 06, 010 (2003).

[10] A.P. Balachandran, S. Kürkçüoğlu, and E. Rojas, J. High Energy Phys. 07, 056 (2002).

[11] D. Klemm, S. Penati, and L. Tamassia, Class. Quantum Grav. 20, 2905 (2003).

[12] R. Casalbuoni, Phys. Lett. 62B, 49 (1976); L. Brink and J. Schwarz, ibid. 100B, 310 (1981).

[13] N. Berkovits, J. High Energy Phys. 09, 016 (2001).

[14] P.A. Grassi, G. Policastro, and P. van Nieuwenhuizen, Phys. Lett. B 553, 96 (2003).

[15] N. Berkovits, Int. J. Mod. Phys. A 16, 801 (2001).

[16] M. Kontsevich, "Deformation quantization of Poisson manifolds, I," q-alg/9709040.

[17] I. Chepelev and C. Ciocarlie, J. High Energy Phys. 06, 031 (2003).

[18] B.L. Cerchiai, J. High Energy Phys. 06, 056 (2003).

[19] I. Bars and C. Deliduman (in preparation). 